\title{
Failure to maintain high-dose treatment regimens during long-term use of zidovudine in patients with symptomatic human immunodeficiency virus type 1 infection
}

\author{
R van Leeuwen, P J van den Hurk, G J Jöbsis, P A van der Wouw, P Reiss, J K M Eeftinck \\ Schattenkerk, S A Danner, J M A Lange
}

\begin{abstract}
Long-term tolerance of zidovudine treatment was retrospectively analysed in 97 patients with AIDS or AIDS-related complex. After one year of treatment $68 \%$ and after two years $87 \%$ of the patients had had at least one dose adjustment during their course of therapy. Myelotoxicity was the most common cause (58\% of all cases) of dose reductions and therapy interruptions (dose adjustments). At the time of the first dose adjustment 33 patients $(34 \%)$ were suffering from anaemia (Hb $<6.0$ g/d1), 20 patients $(21 \%)$ from leukopenia (leukocytes $\left.<1.5 \times 10^{9}\right)$, and 10 patients $(10 \%)$ from thrombocytopenia (thrombocytes $<75 \times 10^{9}$ ). Fifty-six patients $(57 \%)$ needed one or more blood transfusions during therapy. The median time from the start of therapy to the time of the first dose adjustment was 14 (range: 2-64) weeks in patients who had a first dose adjustment because of anaemia without coexisting leukopenia or thrombocytopenia, and 37 (range: 6-85) weeks in patients who had a first dose adjustment because of leukopenia without co-existing anaemia or thrombocytopenia $(p=0 \cdot 01)$. Peripheral blood CD4 positive lymphocyte counts $\leqslant 100 / \mathrm{mm}^{3}$, anaemia, and CDC classification IV-C1 at the start of treatment were associated with a need for an early dose modification or blood transfusion rather than the need for dose modification per se.
\end{abstract}

Department of Internal Medicine (AIDS unit), Academic Medical Centre, University of Amsterdam, Amsterdam, the Netherlands

$R$ van Leeuwen, $P$ J van den Hurk, G J Jöbsis, $P$ A van der Wouw, P Reiss, J K M Eeftinck Schattenkerk, S A Danner

Human Retrovirus Laboratory, Academic Medical Centre, University of Amsterdam, Amsterdam, the Netherlands

J M A Lange
Zidovudine (3'-azido-3'-deoxythymidine, azidothymidine, AZT) is a thymidine analogue that, after intracellular phosphorylation, inhibits the replication of the human immunodeficiency virus type 1 (HIV-1), through interference with reverse transcription. ${ }^{12}$ Initial clinical studies with zidovudine in patients with the acquired immunodeficiency syndrome (AIDS) and AIDS-related complex (ARC) showed that it induced clinical and immunological improvements..$^{3-5}$ In the spring of 1987 the drug was licensed in many countries for the use in patients with severe HIV-1-related disease.

Zidovudine treatment is associated with toxicities which limit its use. ${ }^{6-9}$ In this respect bone marrow toxicity is most important. Anaemia, which can be macrocytic or normocytic is frequently found. After prolonged therapy, leukopenia (primarily attributable to neutropenia) and thrombocytopenia can occur. Bone marrow toxicity has been observed more frequently in patients with pretreatment anaemia, neutropenia, low numbers of peripheral blood CD4 positive lymphocytes or a low serum level of vitamin B12. ${ }^{69}$ Other toxicities seen in patients receiving zidovudine are headaches, nausea, malaise and myalgia. ${ }^{613}$ Myopathy can occur after prolonged treatment. ${ }^{10-1215}$

In a recent study from France, during a treatment period of 31 weeks only $21 \%$ of patients with AIDS tolerated full-dose treatment without a dose reduction or therapy interruption. ${ }^{9}$ We performed a retrospective analysis with a longer follow-up period (up to 147 weeks) in order to determine the long-term tolerance of zidovudine in our patient population with AIDS and ARC.

\section{Methods}

All patients above 18 years of age from our centre who started zidovudine treatment between May 1987 and July 1988 were studied. Those patients in whom the course of zidovudine therapy was not fully documented were subsequently excluded from analysis. Data on the period between the start of 
treatment and the last visit before March 1990, or the time of death were collected from out-patient department records. In most patients the initial dose was 1000 or $1200 \mathrm{mg}$ daily. If there was a need to treat with other myelotoxic therapy, or if there were preexisting haematological abnormalities, the initial dose was 500 or $600 \mathrm{mg}$ daily.

Data were collected regarding: (a) the duration of zidovudine treatment, (b) dose adjustments, (c) haematological data: haemoglobin, leukocytes and thrombocytes at the start of treatment and at the time of dose adjustment, (d) number of peripheral blood CD4 positive lymphocytes at the start of treatment, (e) concomitant use of other potentially myelotoxic therapy: cotrimoxazole, pyrimethamine, interferon, gangciclovir or cytostatic therapy (local or systemic), (f) CDC classification ${ }^{14}$ at the start of treatment.

The following criteria were used to reduce the dose of zidovudine because of myelotoxicity: haemoglobin level below $6.0 \mathrm{~g} / \mathrm{dl}$, and/or (b) leukocyte count below $1 \cdot 5 \cdot 10^{9} / 1$, and/or (c) thrombocyte count below $75 \cdot 10^{9} / 1$.

A change in dosage of zidovudine was considered to be a dose adjustment when a change between the following dose categories was made for a period of more than two weeks: (a) full-dose ( $>600 \mathrm{mg} /$ day), (b) reduced dose ( $\leq 600 \mathrm{mg} /$ day), or (c) interruption of therapy. When patients started with a reduced dose for more than two weeks, this first period also was considered to be a dose adjustment.

The Wilcoxon rank test, the Mann-Whitney U test, and the chi-square test were used for statistical analysis where appropriate.

\section{Results}

During a period of 60 weeks a total number of 104 patients from our centre started treatment with zidovudine for AIDS or ARC. All patients were male, the median age was 39 (range: $24-65$; mean 40) years. Patients were followed for up to 147 weeks. Seven patients were excluded from this analysis: three because they were treated at other centres during a part of the therapy, and four other patients because records were incomplete. In the remaining 97 patients treatment was started at full-dose in 91 cases. In six cases zidovudine was started in a reduced dose, because of concomitant myelotoxic therapy. During follow-up 70 patients $(72 \%)$ died.

The median follow-up period for all patients was 67 (range: $3-147$; mean 68.3 ) weeks. For a median of 32 (range: $0-138$; mean 38.8 ) weeks a full dose of zidovudine was used. It was used in reduced dosage for a median of 8 (range: $0-116$; mean $17 \cdot 8$ ) weeks. In 68 patients a total number of 109 dose reductions was observed. Therapy was interrupted for a median of 5 (range: 0-68; mean 11.7) weeks. In 65 patients a total number of 103 therapy interruptions was observed.

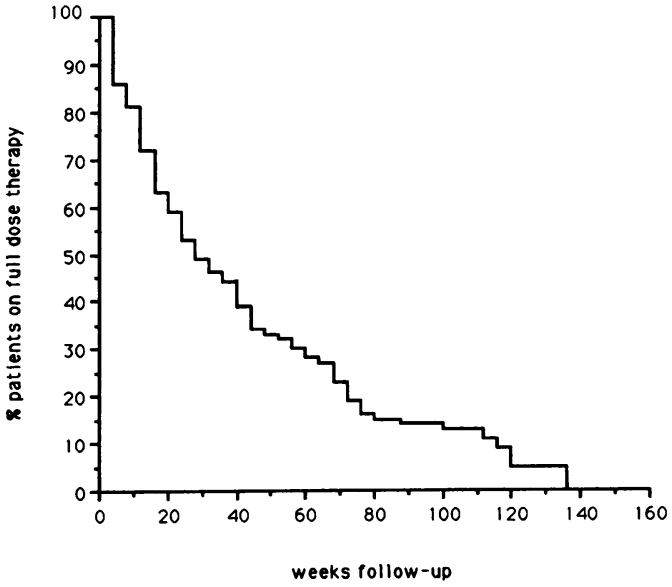

Fig Dose adjustments in full dose therapy because of toxicity or other reasons during 1.36 weeks follow-up (Kaplan-Meier-curve).

The figure shows the incidence of first dose adjustments in the zidovudine treated patients in time. After one year of treatment the cumulative number of patients with dose adjustments (KaplanMeier curve) was $68 \%$, after two years $87 \%$. In those patients who had at least one dose adjustment during their course of therapy, there was a median number of one dose reduction and one therapy interruption.

Table 1 shows the median haematological values at the time of the start of therapy and at the moment of the first dose adjustment. A significant decline in haemoglobin levels and leukocyte counts was observed, while thrombocyte counts did not drop significantly during this period.

Anaemia appeared to be the most common toxicity. In 33 patients ( $34 \%$ of the total population) anaemia was seen at the time of the first dose adjustment. Furthermore, 56 patients $(57 \%)$ needed one or more blood transfusions during therapy. In this group of patients a median number of five (range: 1-21) transfusions was given. The median number of units of packed red blood cells per patient was 11 (range: $2-72$ ). The median number of transfusions in the total population during zidovudine treatment was

Table 1 Median haematological parameters at the start of treatment and at the time of the first dose adjustment

\begin{tabular}{lccl}
\hline & $\begin{array}{l}\text { Start of } \\
\text { treatment }\end{array}$ & $\begin{array}{l}\text { At time of } \\
\text { adjustment }\end{array}$ & \\
\hline $\begin{array}{l}\text { Haemoglobin } \\
(\mathrm{g} / \mathrm{dl})\end{array}$ & $7.6(3.9-10.4)$ & $6.6(2.2-9.7)$ & $\mathrm{p}<0.001$ \\
$\begin{array}{c}\text { Leucocyte count } \\
\left(\cdot 10^{9} / 1\right)\end{array}$ & $3.8(1.6-13.6)$ & $2.2(0.6-6.0)$ & $\mathrm{p}<0.001$ \\
$\begin{array}{c}\text { Platelet count } \\
\left(\cdot 10^{\circ} / 1\right)\end{array}$ & $150(38-335)$ & $135(8-372)$ & n.s. \\
\hline
\end{tabular}

Wilcoxon rank test. n.s. = not significant. 
one (range: 0-21). The median time between the start of therapy and the first blood transfusion was 26 (range: $0-136)$ weeks. In 20 patients $(21 \%)$ the first dose adjustment was made because of leukopenia, while thrombocytopenia was reason for a dose adjustment in 10 patients $(10 \%)$.

Differences in the time of the development of myelotoxicity in distinct cell lines were analysed by comparing the median times from the start of therapy to the moment of first dose adjustment. The median time from the start of therapy to the first dose adjustment was 14 (range: 2-64) weeks in patients $(n=19)$ who had a first dose adjustment because of anaemia without co-existing leukopenia or thrombocytopenia, and 37 (range: 6-85) weeks in patients $(n=9)$ who had a first dose adjustment because of leukopenia without co-existing anaemia or thrombocytopenia ( $p=0.01$, Mann-Whitney $U$ test). In patients with a dose adjustment because of thrombocytopenia $(n=5)$ or myelotoxicity involving more than one cell line $(n=9)$, the median time between the start of treatment and the first dose adjustment was 24 (range: 9-119) weeks. This did not differ significantly from the period found in those who developed solitary anaemia or leukopenia.

The cumulative number of dose adjustments was 212. In 118 cases (56\% of all dose adjustments) dose adjustments were made because of haematological abnormalities. Haemoglobin levels were below $6.0 \mathrm{~g} / \mathrm{dl}$ in 71 cases ( $33 \%$ of all dose adjustments), leukocyte counts below $1 \cdot 5.10^{9} / 1$ in 59 cases $(28 \%$ ) and thrombocyte counts below $75 \cdot 10^{9} / 1$ in 53 cases $(25 \%)$. In 63 cases $(53 \%$ of all dose adjustments because of haematological abnormalities) just one cell line was involved. In 55 cases $(47 \%)$ there was a combination of two or more haematological abnormalities at the time of dose adjustment. There were seven dose adjustments made because of pancytopenia.

Haematological toxicity often recurred in patients who had a dose reduction. When a first adjustment in the therapy was a dose reduction, this was subsequently followed by therapy interruptions in $\mathbf{4 0}$ cases $(71 \%$ of the patients with a dose reduction). A Kaplan-Meier analysis of all patients with a dose reduction as first dose adjustment in the therapy, showed that $40 \%$ were still on zidovudine therapy after 24 weelss, without subsequent stop.

In 59 patients $(70 \%)$ with dose adjustments concomitant potentially myelotoxic therapy was used, in most cases given in low dosages as secondary prophylactic therapy for opportunistic infections.

In 35 patients $(42 \%$ of all patients with dose adjustments) no haematological abnormalities were seen. Dose adjustments in these cases commonly were interruptions of therapy because of subjective side effects such as nausea, or because of terminal disease. In only one case clinically evident myopathy, which was histologically proven, was reason for the discontinuation of therapy.

CDC stage of disease, haemoglobin level, and peripheral blood CD4 positive lymphocyte counts at the start of therapy were tested as predictive factors for toxicity. Table 2 shows that CDC classification IV-C1, haemoglobin $\leq 6.0 \mathrm{mmol} / \mathrm{l}$, and CD4 positive lymphocyte counts $\leq 100 / \mathrm{mm}^{3}$ were associated with the occurrence of an early dose modification and/or an early blood transfusion. There were no significant differences between groups in the number of patients that eventually developed toxicity.

\section{Discussion}

This analysis shows that only a minority of patients with severe symptomatic HIV-1-related disease can tolerate full-dose zidovudine regimens for prolonged periods. After one year of treatment $68 \%$ and after two years $87 \%$ of the patients had had at least one dose adjustment during their course of therapy (Kaplan- Meier-curve). These findings are comparable with the rate found in the extended follow-up from the original American phase II study. ${ }^{15}$

Table 2 Factors predictive for dose adjustment during the course of therapy

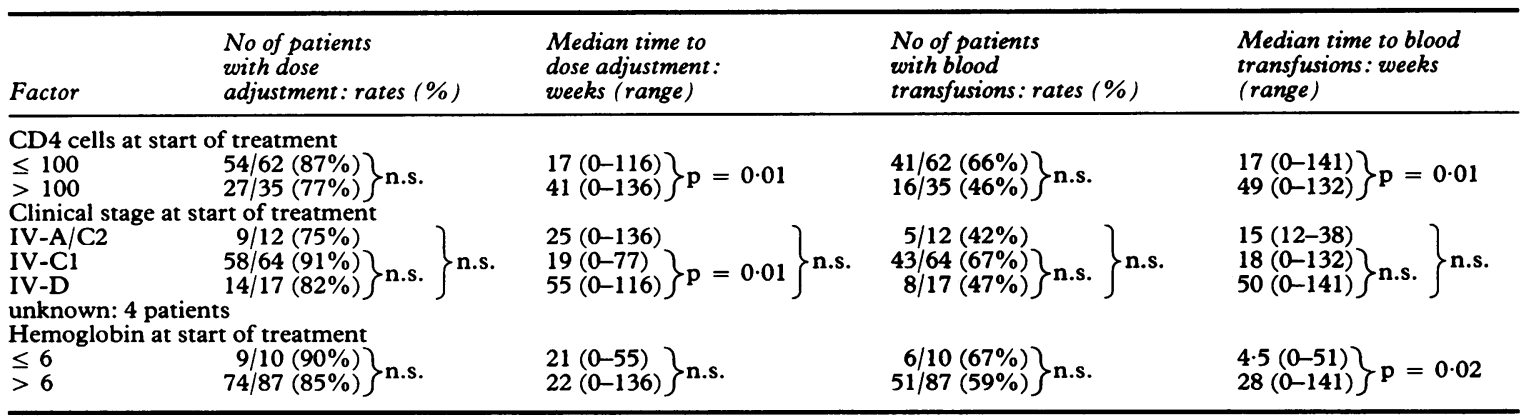

Differences in time were compared with a Mann-Witney U test, differences in rates were compared with a chi-square test. n.s. = not significant. 
Dose reductions and discontinuation of therapy were commonly due to haematological toxicity, as reported previously. ${ }^{69}$ Anaemia was the most frequent cause for dose adjustments, followed by leukopenia (which has previously been found to be primarily attributable to neutropenia. ${ }^{6}$ The decline in haemoglobin levels and white blood cell counts from their initial values at the start of treatment until the moment of the first dose adjustment was more pronounced than the decline observed in the thrombocyte count, the latter not reaching a statistical significance. This confirms that the myelotoxic effect of zidovudine especially concerns the red and white cell lines. Thrombocyte counts may even rise during zidovudine therapy. ${ }^{615-18}$

Anaemia was an early sign of toxicity compared to leukopenia. The median time to the development of anaemia was 14 weeks, while leukopenia occurred after a median of 37 weeks of therapy. This difference was significant. Myelotoxicity involving more than one cell line occurred after a median of 24 weeks. This period did not appear to be significantly different from the time to the occurrence of anaemia or from the time to the occurrence of leukopenia. As reported in earlier studies, ${ }^{69}$ peripheral blood CD4 positive lymphocyte counts below or equal to $100 / \mathrm{mm}^{3}$, anaemia, and CDC classification IV-Cl at the start of treatment were associated with poor tolerance of full dose zidovudine treatment. Our study, with a longer follow-up, shows that these markers predict a need for an early dose modification or blood transfusion rather than the need for dose modification per se.

Concomitant potential myelotoxic medicine was often used (in $70 \%$ of the patients). In most cases it was given as low dose prophylactic therapy for opportunistic infections, which makes it unlikely that this played a major role in the observed toxicity. Although dose reductions were followed by subsequent therapy interruptions in a majority of cases, zidovudine treatment could often be continued for an extended period thereafter; $40 \%$ of the patients were still on the drug a half year after a first reduction.

Haematological toxicity was the most common reason for a dose modification. In 35 patients $(42 \%$ of all dose adjustments) however, zidovudine was stopped for other reasons, such as nonhaematological side effects or terminal disease. Myopathy has been reported to occur in an increasing number of patients on long-term zidovudine therapy. ${ }^{10-12} 15$ In this study only one case of histologically proven myopathy was seen, although 54 patients did receive zidovudine for more than a year.

Thus, the majority of patients with AIDS or ARC who are treated with a high dose of zidovudine, at this moment the sole antiretroviral drug which is licensed, cannot be maintained on these regimens, most commonly due to the development of haematological toxicity. Up until now the established zidovudine starting dose in many countries has been 1000-1500 mg per day. Treatment with lower daily doses of zidovudine may lead to reduced toxicity, while maintaining efficacy. ${ }^{13}$ It is, however, as yet unknown what influence such low-dose regimens will have on the rate at which viral drug resistance develops. ${ }^{19-21}$ Treatment with cytokines, such as granulocyte- and granulocyte-macrophage colony stimulating factors and erythropoietin, may be another promising option to counter zidovudineinduced myelotoxicity. ${ }^{22-24}$

Address for correspondence: Dr J M A Lange, Department of Internal Medicine, Academic Medical Centre, University of Amsterdam, Room F5-163, Meibergdreef 9, 1105 AZ Amsterdam, the Netherlands.

1 Mitsuya $\mathrm{H}$, Weinhold KJ, Furman PA, et al. 3'-azido-3'-deoxythymidine (BW A509U): An antiviral agent that inhibits the infectivity and cytopathic effect of human T-lymphotropic virus type III/lymphadenopathy-associated virus in vitro. Proc Natl Acad Sci USA 1985;82:7096-100.

2 Furman PA, Fyfe JA, St Clair MH, et al. Phosphorylation of 3'azido- $3^{\prime}$-deoxythymidine and selective interaction of the $5^{\prime}$ triphosphate with human immunodeficiency virus reverse transcriptase. Proc Natl Acad Sci USA 1986;83:8333-7.

3 Yarchoan R, Klecker RW, Weinhold KJ, et al. Administration of 3'-azido-3'-deoxythymidine, an inhibitor of HTLV III/ LAV replication, to patients with AIDS or AIDS related complex. Lancet 1986;i:575-80.

4 Fischl MA, Richman DD, Grieco MH, et al. The efficacy of azidothymidine (AZT) in the treatment of patients with AIDS and AIDS-related complex: a double-blind, placebo-controlled trial. N Engl J Med 1987;317:185-91.

5 Yarchoan R, Berg G, Brouwers P, et al. Response of humanimmunodeficiency-virus-associated neurological disease to 3'azido-3'-deoxy thymidine. Lancet 1987;i:132-5.

6 Richman DD, Fischl MA, Grieco MH, et al. The toxicity of azidothymidine $(A Z T)$ in the treatment of patients with AIDS and AIDS-related complex: a double blind placebo-controlled trial. N Engl J Med 1987;317:192-7.

7 Gill PS, Rarick M, Brynes RK, Causey D, Loureiro C, Levine AM. Azidothymidine associated with bone marrow failure in the acquired immunodeficiency syndrome (AIDS). Ann Intern Med 1987;107:502-5.

8 Walker RE, Parker RI, Kovacs JA, et al. Anaemia and erythropoiesis in patients with the acquired immunodeficiency syndrome (AIDS) and Kaposi sarcoma treated with zidovudine. Ann Intern Med 1988;108:372-6.

9 Dournon E, Matheron S, Rozenbaum W, et al. Effects of zidovudine in 365 consecutive patients with AIDS and AIDSrelated complex. Lancet 1988;ii:1297-302.

10 Helbert M, Fletcher T, Peddle B, Harris JRW, Pinching AJ. Zidovudine-associated myopathy. Lancet 1988;ii:689-90.

11 Fischl MA, Gagnon S, Uttamachandani R, et al. Myopathy associated with long-term zidovudine therapy (abstract No MBP 329). Abstracts of the Vth International Conference on AIDS, Montreal 1989.

12 Dalakas MC, Illa I, Pezeshkpour GH, Laukaitis JP, Cohen B, Griffin JL. Mitochondrial myopathy caused by long-term zidovudine therapy. N Engl J Med 1990;322:1098-105.

13 Fischl MA. State of antiretroviral therapy with zidovudine. AIDS 1989;3 (Suppl 1): S137-43.

14 Centers for Disease Control. Classification system for human $T$ lymphotropic virus type-III lymphadenopathy-associated virus infection. MMWR 1986;35:334-9.

15 Fischl MA, Richman DD, Causey DM, et al. Prolonged zidovudine therapy in patients with AIDS and advanced AIDS-related complex. JAMA 1989;262:2405-10.

16 Hymes KB, Greene JB, Karpatkin S. The effect of azidothymidine on HIV-related thrombocytopenia. N Engl J Med 1988;318:516-7. 
17 Swiss Group for Clinical Studies on the Acquired Immunodeficiency Syndrome (AIDS). Zidovudine for the treatment of thrombocytopenia associated with human immunodeficiency virus (HIV); a prospective study. Ann Intern Med 1988;109:718-21.

18 Hollak CEM, Kersten MJ, van der Lelie J, Lange JMA. Thrombocytopenic purpura as first manifestation of human immunodeficiency virus type I (HIV-1) infection Netherl $J$ Med 1990;37:63-8.

19 Larder BA, Darby G, Richman DD. HIV with reduced sensitivity to zidovudine (AZT) isolated during prolonged therapy. Science 1989;243:1731-4.

20 Larder BA, Kemp SD. Multiple mutations in HIV-1 reverse transcriptase confer high-level resistance to zidovudine (AZT). Science 1989;246:1155-8.

21 Land S, Treloar G, McPhee D, et al. Decreased in vitro susceptibility to zidovudine of HIV isolates obtained from patients with AIDS. J Infect Dis 1990;161:326-9.
22 Van Leeuwen R, van der Wouw PA, Lange JMA, Danner SA. Treatment with G-CSF in zidovudine treated HIV infected patients with leukopenia. Abstracts of the meeting of the Dutch society for Internal Medicine, Veldhoven 1990.

23 Groopman JE, Mitsuyasu RT, DeLo MJ, Oette DH, Golde $\mathrm{DW}$. Effect of recombinant human granulocyte-macrophage colony-stimulating factor on myelopoiesis in the acquired immunodeficiency syndrome. N Engl J Med 1987;317:593-8.

24 Miles S, Mitsuyasu R, Fink M, et al. Recombinant granulocyte colony stimulating factor ( $r$-metHuG-CSF) and recombinant erythropoietin ( $\mathbf{r}$-HuEPO) may abrogate the neutropenia and anemia of AIDS and may allow for resumption of zidovudine (AZT) in patients with AIDS (abstract No MCP 52). Abstracts of the Vth International Conference on AIDS, Montreal 1989.

Accepted for publication 3 September 1990 